\title{
Electron cooling of high-energy protons in a multiring trap with a tank circuit monitoring the electron-plasma oscillations
}

\author{
H. Higaki, ${ }^{*}$ N. Kuroda, T. Ichioka, ${ }^{*}$ K. Yoshiki Franzen, Z. Wang, K. Komaki, and Y. Yamazaki ${ }^{\dagger}$ \\ Institute of Physics, University of Tokyo, 3-8-1, Komaba, Meguro, Tokyo 153-8902, Japan \\ M. Hori \\ CERN, CH-1211 Geneva 23, Switzerland
}

N. Oshima and A. Mohri

RIKEN, 2-1 Hirosawa, Wako, Saitama 351-0198, Japan

(Received 29 October 2001; published 2 April 2002)

\begin{abstract}
Electron cooling of energetic protons in a multiring trap was investigated experimentally with a tank circuit monitoring electron-plasma oscillations in the trap. The energy of protons was determined by time-of-flight measurements. It is found that a simple model can explain the qualitative behavior of both electron and proton energy when the initial energy of protons is less than $2 \mathrm{keV}$. Monitoring the electron-plasma temperature with a tank circuit can be an effective tool when energetic particles are electron cooled in a multiring trap.
\end{abstract}

DOI: 10.1103/PhysRevE.65.046410

PACS number(s): 52.27.Jt, 52.35.Fp, 52.20.Fs

\section{INTRODUCTION}

Electron-cooling techniques have found various experimental applications such as in accelerators and cooling of high-energy particles in a trap. Production of ultraslow antiprotons was made possible with the combination of a degrader foil and electron cooling in a Penning trap [1]. Positron cooling of highly charged ions (HCI) in a multiring trap is in progress to produce a low-energy HCI beam. These low-energy antiprotons and HCI beams will open new research fields [2]. Although the electron cooling of lowenergy protons $(\leqslant 80 \mathrm{eV})$ in the nested Penning trap was studied [3], little has been reported on the process to cool particles having higher energies. This is particularly true for $\sim 1 \mathrm{keV}$ or higher energy particles, which is discussed in the present paper.

For understanding the electron-cooling process in the trap, it is important to observe signals from the trapped charged particles during the process. Fortunately, various techniques developed for a small number of charged particles in a Penning trap are also applicable to a large number of charged particles (a plasma) in a multiring trap. A tank circuit can be an especially powerful tool for a nondestructive diagnosis of trapped charged particles. A large number of cold electrons used to cool high-energy protons in the trap are regarded as a spheroidal nonneutral electron plasma. The dispersion relation for the electrostatic oscillations of a cold spheroidal nonneutral plasma was derived by Dubin [4]. With the dispersion relation, the fundamental harmonic oscillation $(l=1$ mode) is derived easily. It is known that the number of trapped

\footnotetext{
*Present address: Plasma Research Center, University of Tsukuba, 1-1-1 Tennoudai, Tsukuba, Ibaraki, 305-8571 Japan.

**Present address: Institute of Physics and Astronomy, Aarhus University, Ny Munkegade Bygn.520 DK8000, Aarhus C, DENMARK.

${ }^{\dagger}$ Also at RIKEN, 2-1 Hirosawa, Wako, Saitama 351-0198, Japan.
}

charged particles can be determined with a tank circuit monitoring the $l=1$ mode $[5,6]$. The second order axial oscillation $(l=2$ mode) is interesting since its frequency depends on the electron energy (or electron-plasma temperature) $[7,8]$.

Here, the purpose of the experiment is to investigate the cooling process of energetic protons by monitoring the $l$ $=2$ mode of the electron plasma that cool high-energy protons in a multiring trap. Since the signal from high-energy protons in a trap is difficult to observe, the signal from electrons is used to probe the behavior of protons.

\section{EXPERIMENTAL SETUP}

To accomplish the experimental purpose mentioned above, a multiring trap was adopted [9]. The inner diameter of the electrodes is $2 b=4 \mathrm{~cm}$ and the axial length $L_{0}$ for confining high-energy protons is $\sim 30 \mathrm{~cm}$. By applying proper voltages on each electrode, this multiring electrode trap can provide an electrostatic quadrupole potential

$$
\phi(\rho, z)=-V\left(\rho^{2}-2 z^{2}\right) /\left(2 L^{2}+b^{2}\right)
$$

in the cylindrical coordinates $(\rho, \theta, z)$ with the axial dimension $2 L=12.3 \mathrm{~cm}$. The trap was immersed in a uniform axial magnetic field $B=10 \mathrm{kG}$. Since the vacuum should be good enough to avoid collisions between high-energy protons and background residual gases, the chamber wall that contains the trap was cooled lower than $10 \mathrm{~K}$ with continuous flow of liquid He. The vacuum pressure measured outside the cold region was $\leqslant 2 \times 10^{-10}$ torr. A schematic drawing of the experimental setup is shown in Fig. 1.

High-energy protons $(1 \sim 4 \mathrm{keV})$ were provided with a duoplasmatron ion source via a magnetic analyzer, which selects and transports protons to the trap.

Electrons with a typical energy of $\sim 60 \mathrm{eV}$ were provided by a field emitter array that was placed where the field strength was $\sim 100 \mathrm{G}$ and $5 \mathrm{~cm}$ off from the axis of the 


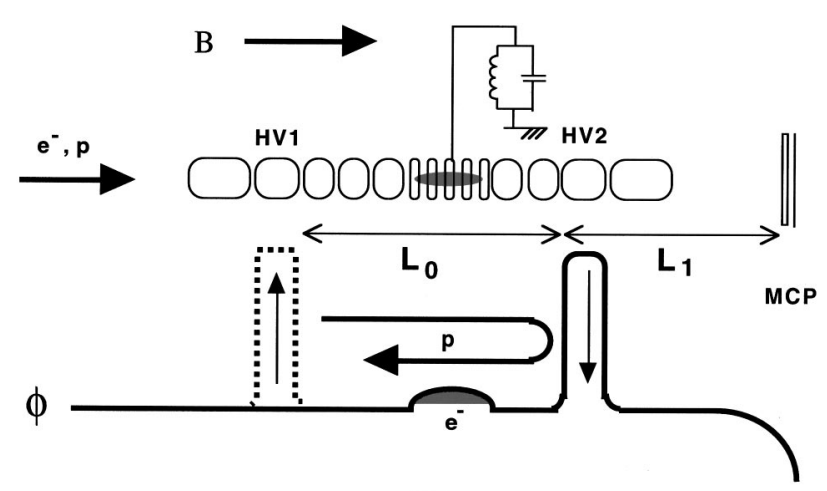

$1 \mathrm{~T}$

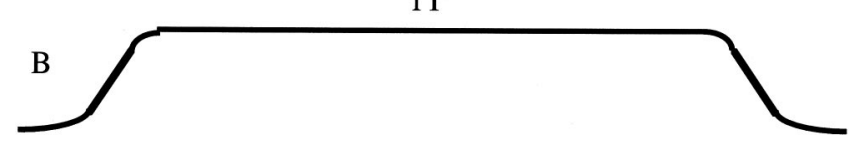

FIG. 1. A schematic drawing of the experimental setup.

magnetic field and the trap so that the high-energy protons could be injected into the trap. The number of electrons stored in the trap can be easily controlled by varying the injection time of electrons. Here, $10^{8}$ electrons were confined routinely with the confinement time longer than $1000 \mathrm{sec}$. To detect the $l=2$ mode of the electron plasma, a tank circuit composed of a tunable capacitor and an inductor was attached to the ring electrode at the center. The signal was detected with a fast fourier transform spectrum analyzer.

A microchannel-plate (MCP) of $7 \mathrm{~cm}$ in diameter was installed on the other side of the magnet, where the field strength was $\sim 100 \mathrm{G}$, to detect high-energy protons $(50-$ $4000 \mathrm{eV}$ ) by time-of-flight (TOF) measurement. The length $L_{1}$ from the end of the trap region to the MCP is $\sim 110 \mathrm{~cm}$. Thus, the length of the trap should be considered when TOF spectra are evaluated.

The experimental procedure is as follows. First, proper electrostatic voltages are applied on each electrode to confine electrons in the quadrupole potential with $V=-50 \mathrm{~V}$ in Eq. (2.1). After the electrons are accumulated in the trap, a high voltage is applied to the electrode HV2(exit) to reflect back the injected protons. Then, a few $\mathrm{keV}$ protons are injected and the voltage on the electrode HV1 (entrance) is switched on to confine protons. After waiting for a certain interval of time with monitoring the electron-plasma oscillation through a tank circuit, the electrode HV2 is grounded to detect the energy of protons with the MCP.

\section{RESULTS AND DISCUSSIONS}

The TOF signals of confined high-energy protons detected with the MCP are shown in Fig. 2. In Fig. 2(a), 2-keV protons $\left(\sim 5 \times 10^{5}\right)$ are confined without electrons. The solid and broken lines are the TOF spectra of protons confined for 10 and $80 \mathrm{sec}$, respectively. The confinement time defined as the time for the trapped particle number to be half of the original value is estimated to be about $80 \mathrm{sec}$ in case of $2-\mathrm{keV}$ protons. It is noted that the peak position of the TOF spectrum does not depend on the trapping time, i.e., the proton energy stays the same. Therefore, the energy loss of protons through the collisions with background gas is negligibly
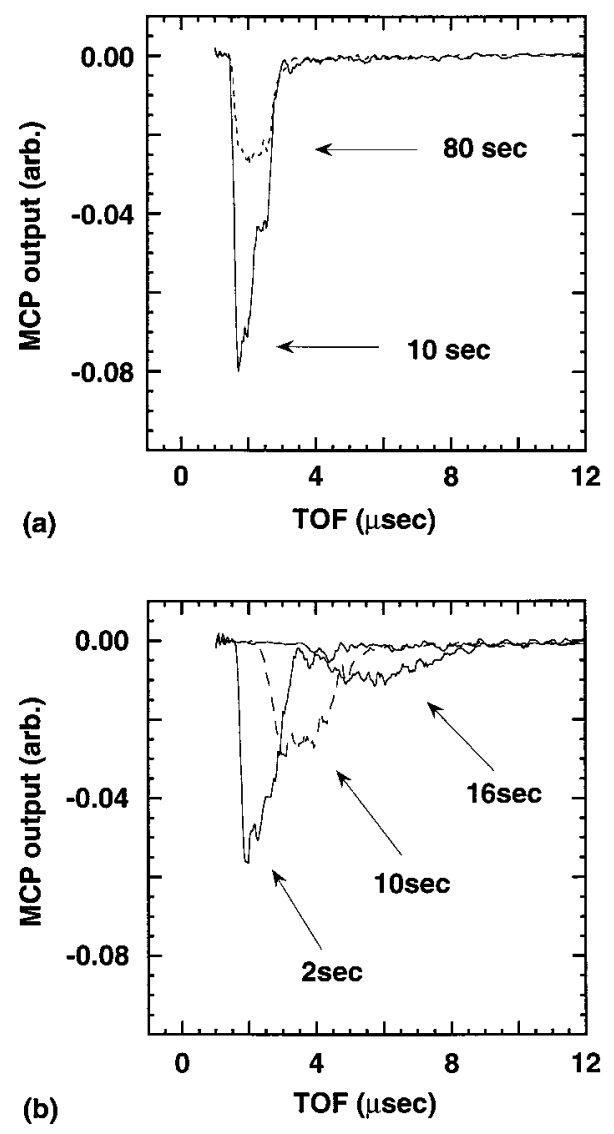

FIG. 2. (a) TOF signals of $2-\mathrm{keV}$ protons without electrons. The solid and dashed lines correspond to the trapping time of 10 and 80 sec, respectively. (b) TOF signals of protons with electrons. The trapping times are 2,10 , and $16 \mathrm{sec}$, respectively.

small. The pulse width of about $1.5 \mu \mathrm{sec}$ mainly reflects the length of the trap in this case. Shown in Fig. 2(b) are the TOF signals of confined protons with $1.0 \times 10^{8}$ electrons. The trapping times are 2, 10, and $16 \mathrm{sec}$, respectively. It is clearly seen that the proton energy $E_{p}$ decreases as the trapping time gets longer. The maximum energy $E_{\max }$ and minimum energy $E_{\min }$ of protons are easily obtained from each TOF spectrum. For evaluating the mean energy of confined protons, a shifted Maxwellian distribution function and a uniform spatial distribution of protons inside the trap region are assumed to reconstruct an obtained TOF spectrum. The mean energy of confined protons are 1860, 780, and $280 \mathrm{eV}$ at 2, 10, and $16 \mathrm{sec}$, respectively. Similar measurements are performed with the proton injection energy of 1, 2, 3, and $4 \mathrm{keV}$.

A cloud of electrons to cool energetic protons inside the trap behaves as a nonneutral plasma. Its electrostatic oscillation frequency $f_{l}=\omega_{l} / 2 \pi$ with the axial mode number $l$ is estimated from Dubin's dispersion relation [4]. Introducing the electron-plasma frequency $\omega_{p}=\sqrt{4 \pi n_{e} e^{2} / m}$ and the electron cyclotron frequency $\Omega_{c}=e B / m c$ with the electron density $n_{e}$, charge $e$ and mass $m$, the rigid rotation frequency of the nonneutral electron plasma is given by $\omega_{r} \sim \omega_{p}^{2} / 2 \Omega_{c}$. Under the conditions $\omega_{p} \ll \Omega_{c}$ and $\omega_{r} \ll \omega_{l} \ll \Omega_{c}$, the dispersion relation is approximated by [7] 


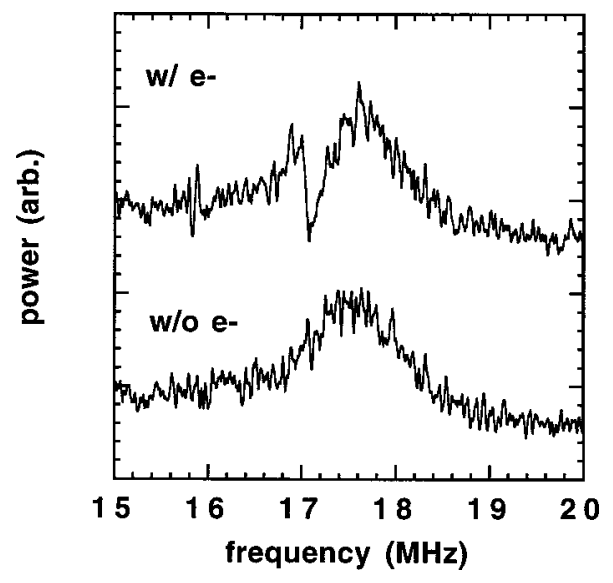

FIG. 3. Examples of tank circuit signals with and without electrons.

$$
\epsilon_{3}-\frac{k_{2}}{k_{1}} \frac{P_{l}\left(k_{1}\right) Q_{l}^{\prime}\left(k_{2}\right)}{P_{l}^{\prime}\left(k_{1}\right) Q_{l}\left(k_{2}\right)}=0 .
$$

Here, $k_{1}=\alpha\left(\alpha^{2}-\epsilon_{3} / \epsilon_{1}\right)^{-1 / 2}, \quad k_{2}=\alpha\left(\alpha^{2}-1\right)^{-1 / 2}, \quad \epsilon_{3}=1$ $-\omega_{p}{ }^{2} / \omega^{2}$, and $\epsilon_{1}=1-\omega_{p}{ }^{2} /\left(\omega^{2}-\Omega_{c}{ }^{2}\right) \sim 1 . P_{l}$ and $Q_{l}$ are Legendre functions of the first and second kinds. The parameter $\alpha$ is the aspect ratio of a spheroidal plasma defined as the ratio of the axial length to the diameter. Using Eq. (3.1), the frequency of the $l=1$ mode is given by $f_{1}=\sqrt{4 e V / m\left(2 L^{2}+b^{2}\right)} / 2 \pi \sim 10.5 \mathrm{MHz}$ with the present

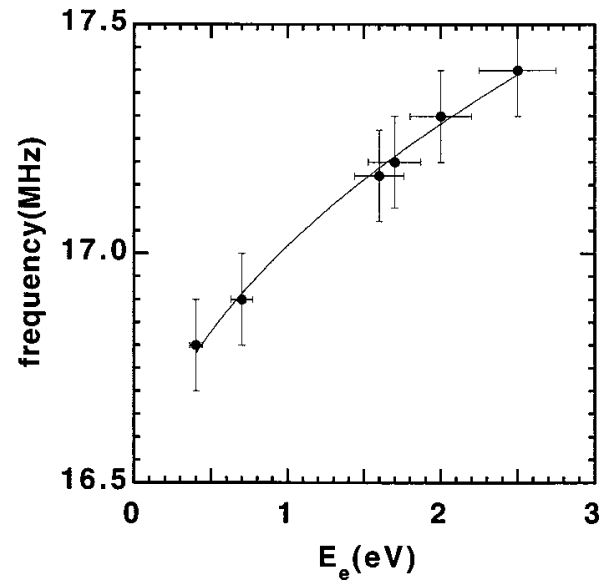

FIG. 4. Calibration of the $l=2$ mode frequency of the electron plasma against its electron energy.

experimental parameters. The oscillation frequency of the $l=2$ mode is $f_{2} \sim 16.4 \mathrm{MHz}$ with an electron density $n_{e}$ $\sim 3.5 \times 10^{7} \mathrm{~cm}^{-3}$. As mentioned in the preceding section, a tank circuit with the resonance frequency near $f_{2}$ is attached to one of the ring electrodes to detect the electron-plasma oscillation. Examples of power spectra are shown in Fig. 3 in logarithmic scale. The resonance spectrum of the tank circuit without electrons is shown at the bottom. At the top, the spectrum with electrons is shown, which is shifted upward for the clarity. Here, the $l=2$ mode is detected as a dip in the spectrum and the resonance frequency $f_{2}$ is defined as the

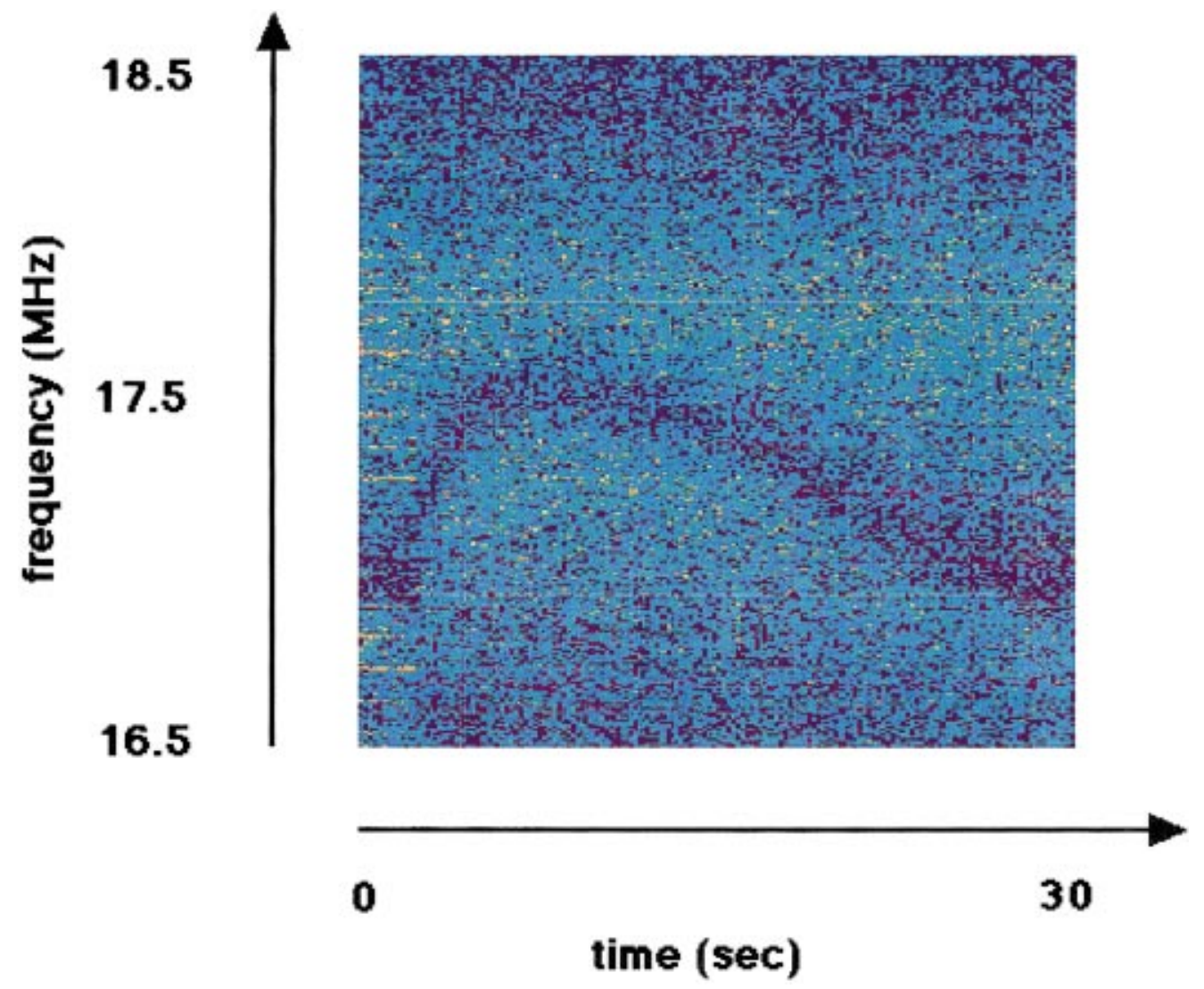

FIG. 5. (Color) A tank circuit signal after the injection of 2-keV protons. 


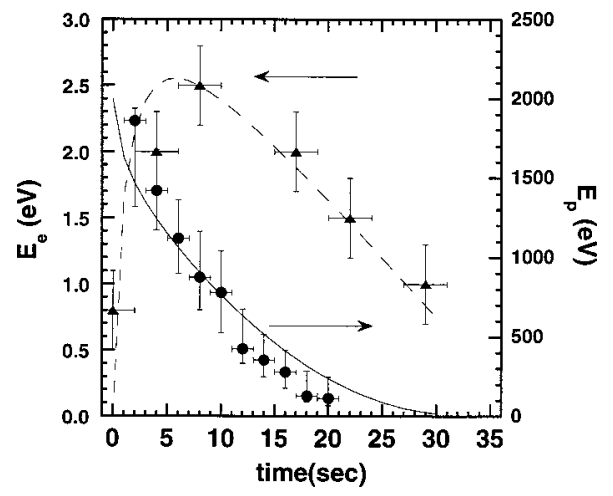

FIG. 6. Calculated proton energy (solid line) and electron energy (dashed line) as functions of time. Measured values are also plotted.

bottom of the dip. The resonance frequency depends on the electron-plasma temperature and the dip shifts higher when the plasma temperature becomes higher. Also, when the plasma occupies the large volume inside the trap, the observed resonance frequency is shifted due to the image charge effect $[7,8]$. With the current experimental parameters, the frequency $f_{2}$ is affected by the image charge. Therefore, the frequency shifts caused by the plasma temperature have to be measured at a fixed total electron number $N_{e}$. In Fig. 4, observed plasma oscillation frequencies are plotted against electron energy $E_{e}$ for $N_{e} \sim 1.0 \times 10^{8}$. Assuming a Maxwell distribution, the electron energy is determined by the number of electrons escaping from the trap when the potential on the electrode is changed $[10,11]$. Less than $0.5 \%$ of the total electrons are used for the measurement to reduce the space charge effect. Typical parameters of the electron plasma for the series of the experiments are the total electron number $N_{e} \sim 1.0 \times 10^{8}, \quad$ electron density $n_{e} \sim 3.5$ $\times 10^{7} \mathrm{~cm}^{-3}$, aspect ratio $\alpha \sim 6.5$, and Debye length $\lambda_{D}$ $\sim 0.13 \mathrm{~cm}$ at $E_{e} \sim 1 \mathrm{eV}$.

Shown in Fig. 5 is the observed frequency $f_{2}$ as a function of time. Since the frequency shift of $f_{2}$ is calibrated against $E_{e}$ in Fig. 4, the electron energy can be monitored while high-energy protons are cooled with electrons in the trap. The initial frequency $f_{2} \sim 16.8 \mathrm{MHz}$, which corresponds to the electron energy of $\sim 0.3 \mathrm{eV}$, increases abruptly after the injection of high-energy protons ( $2 \mathrm{keV}$ in this case), and reaches the maximum $\sim 17.4 \mathrm{MHz}$ within several seconds, which is about $2.5 \mathrm{eV}$ in the electron energy. Then, it falls off gradually with the time scale longer than the synchrotron radiation cooling time $\tau_{r} \sim 6 \times 10^{8} / B^{2} \mathrm{sec}$. This synchrotron radiation cooling time $\tau_{r}$ is estimated with the present experimental setup by observing the cooling of a hot electron plasma without high-energy protons. To evaluate the interaction between electrons and protons, a simple model is considered [12] with the experimental parameters $n_{e} \sim 3.5$ $\times 10^{7} \mathrm{~cm}^{-3}, N_{e} \sim 1.0 \times 10^{8}$, and $N_{p} \sim 5 \times 10^{5}$. Assuming that the energy of protons is distributed equally to all the electrons, the following differential equations can be solved numerically:

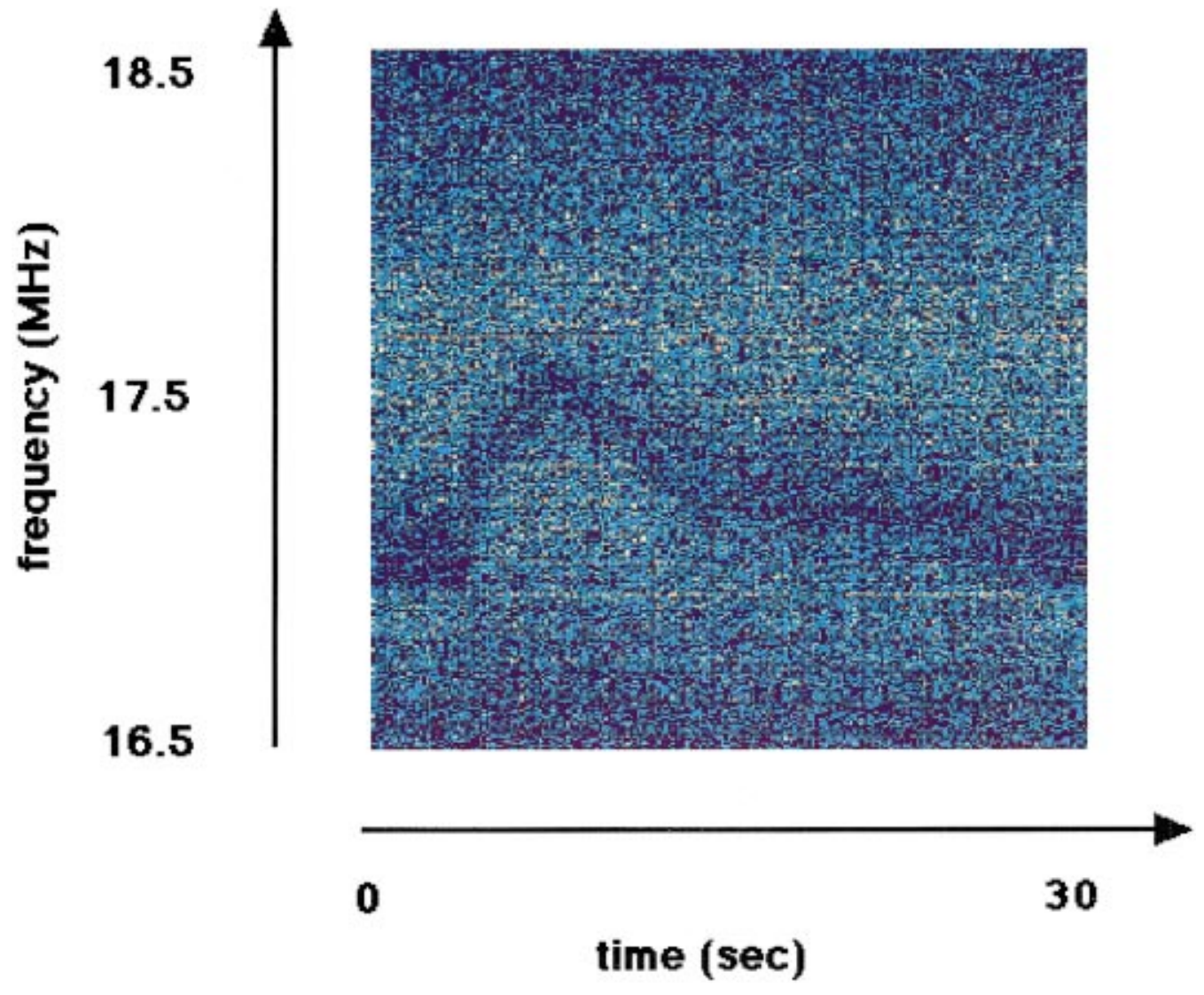

FIG. 7. (Color) A tank circuit signal after the injection of 4-keV protons. 


$$
\begin{aligned}
\frac{d E_{p}}{d t}= & -0.2 \frac{4 \pi n_{e} e^{4}}{v_{p} m_{e}} \ln \Lambda\left[\operatorname{erf}\left(\frac{v_{p}}{v_{e}}\right)-\frac{2}{\sqrt{\pi}} \frac{v_{p}}{v_{e}}\right. \\
& \left.\times\left(1+\frac{m_{e}}{m_{p}}\right) \exp \left(-\frac{v_{p}^{2}}{v_{e}^{2}}\right)\right], \\
\frac{d E_{e}}{d t}= & 0.2 \frac{N_{p}}{N_{e}} \frac{4 \pi n_{e} e^{4}}{v_{p} m_{e}} \ln \Lambda\left[\operatorname{erf}\left(\frac{v_{p}}{v_{e}}\right)-\frac{2}{\sqrt{\pi}} \frac{v_{p}}{v_{e}}\right. \\
& \left.\times\left(1+\frac{m_{e}}{m_{p}}\right) \exp \left(-\frac{v_{p}^{2}}{v_{e}^{2}}\right)\right]-\frac{E_{e}}{\tau_{r}} .
\end{aligned}
$$

Here, the Coulomb logarithm is denoted by $\ln \Lambda$ $=\ln \left\{\lambda_{D} /\left[e^{2} / m_{e}\left(v_{e}+v_{p}\right)^{2}\right]\right\}$ with $v_{e}$ and $v_{p}$, the thermal velocity of electrons and protons. A coefficient 0.2 is introduced since protons interact with electrons only when they travel through the electron plasma. The axial length of the electron plasma is estimated to be $\sim 6 \mathrm{~cm}$. Shown in Fig. 6 are the calculated proton energy (solid line) and electron energy (dashed line) as functions of time. The injection energy of protons is $2 \mathrm{keV}$. Also shown are the experimental proton energy and electron energy during the electron cooling of 2 $\mathrm{keV}$ protons. The experimental proton energy (solid circle) is the mean energy estimated from a TOF signal. For error bars, $E_{\max }$ and $E_{\text {min }}$ are used. The experimental electron energy (solid triangle) is estimated from the observed $f_{2}$ frequency shift and its calibration shown in Fig. 4. It is seen that the overall behavior is well reproduced, although the experimental proton energy decreases slightly faster than the calculated one. This simple model can explain qualitatively the interaction between electrons and protons when the proton injection energy is less than $2 \mathrm{keV}$.

However, when the injected proton energy is higher than $3 \mathrm{keV}$, the qualitative behavior of $f_{2}$ frequency shift changes.
Although the resonance frequency $f_{2}$ increases in the same time scale (several seconds), it falls off more quickly. In this case, the frequency $f_{2}$ falls off within several seconds. Then, $f_{2}$ keeps a slightly higher frequency and returns to the original value. The temporal behavior of $f_{2}$ with $4-\mathrm{keV}$ protons is shown in Fig. 7. Unfortunately, the simple model cannot explain the qualitative behavior of the process. Although no proper experimental evidence was found with the present setup, high-energy protons might interact with a part of electrons and the local heat up of electrons might lead to a deformation of the spheroidal plasma. Or the beam plasma instability might result in the large perturbation of the plasma. The velocity of $4-\mathrm{keV}$ protons is close to the estimated phase velocity of the $l=2$ mode $\left(1 \times 10^{8} \mathrm{~cm} / \mathrm{sec}\right)$. Since it is important to understand the electron cooling of high-energy protons for the effective cooling of high-energy particles, it should be investigated in future experiments.

\section{SUMMARY}

A tank circuit monitoring the $l=2$ mode of the electron plasma is applied for the first time to detect the signal during the electron cooling of high-energy protons in a multiring trap. The frequency shift of the monitored oscillation is qualitatively explained with a simple model when the energy of protons is less than $2 \mathrm{keV}$. This method will be employed by the ASACUSA experiment at AD, CERN.

\section{ACKNOWLEDGMENTS}

The authors acknowledge the support of the Cryogenic Center at Komaba, University of Tokyo. This work is supported by the Grant-in-Aid for Creative Basic Research (10NP0101), Ministry of Education, Science, and Culture. Authors H.H., Z.W., K.Y.F., and M.H. are also supported by JSPS.
[1] G. Gabrielse, X. Fei, L. A. Orozco, S. L. Rolston, R. L. Tjoelker, T. A. Trainor, J. Haas, H. Kalinowsky, and W. Kells, Phys. Rev. Lett. 63, 1360 (1989).

[2] Y. Yamazaki, Nucl. Instrum. Methods Phys. Res. B 154, 174 (1999).

[3] D. S. Hall and G. Gablielse, Phys. Rev. Lett. 77, 1962 (1996).

[4] D. H. E. Dubin, Phys. Rev. Lett. 66, 2076 (1991).

[5] D. J. Wineland and H. G. Dehmelt, J. Appl. Phys. 46, 919 (1975).

[6] X. Feng, M. Charlton, M. Holtzscheiter, R. A. Lewis, and Y. Yamazaki, J. Appl. Phys. 79, 8 (1996).

[7] M. D. Tinkle, R. G. Greaves, C. M. Surko, R. L. Spencer, and G. W. Mason, Phys. Rev. Lett. 72, 352 (1994).
[8] H. Higaki and A. Mohri, Jpn. J. Appl. Phys., Part 1 36, 5300 (1997).

[9] A. Mohri, H. Higaki, H. Tanaka, Y. Yamazawa, M. Aoyagi, T. Yuyama, and T. Michishita, Jpn. J. Appl. Phys., Part 1 37, 664 (1998).

[10] A. W. Hyatt, C. F. Driscoll, and J. H. Malmberg, Phys. Rev. Lett. 59, 2975 (1987).

[11] D. L. Eggleston, C. F. Driscoll, B. R. Beck, A. W. Hyatt, and J. H. Malmberg, Phys. Fluids B 4, 3432 (1992).

[12] D. V. Sivukhin, in Reviews of Plasma Physics, edited by Acad. M. A. Leontovich (Consultants Bureau, New York, 1966), Vol. 4. 